\title{
Morbidity of fungal infections caused by Malassezia furfur in neonatal intensive care units
}

\section{Priputnevich T.V.,Rodchenko J.V., Lyubasovskaya L.A., Dubodelov D.V., Zubkov, V.V., Isaeva E.L.,Gordeev A.B., lonov O.V., Nikitina I.V., Burov A.A., Podurovskaya J.L., Sukhikh G.T.}

National Medical Research Center for Obstetrics, Gynecology and Perinatology named after Academician V.I.Kulakov of Ministry of Healthcare of Russian Federation

4, Oparin street, Moscow, Russian Federation, 117997

E-mail: priput1@gmail.com

Objectives: To study the morbidity of fungal infections caused by Malassezia furfur (M. furfur) in neonatal intensive care units.

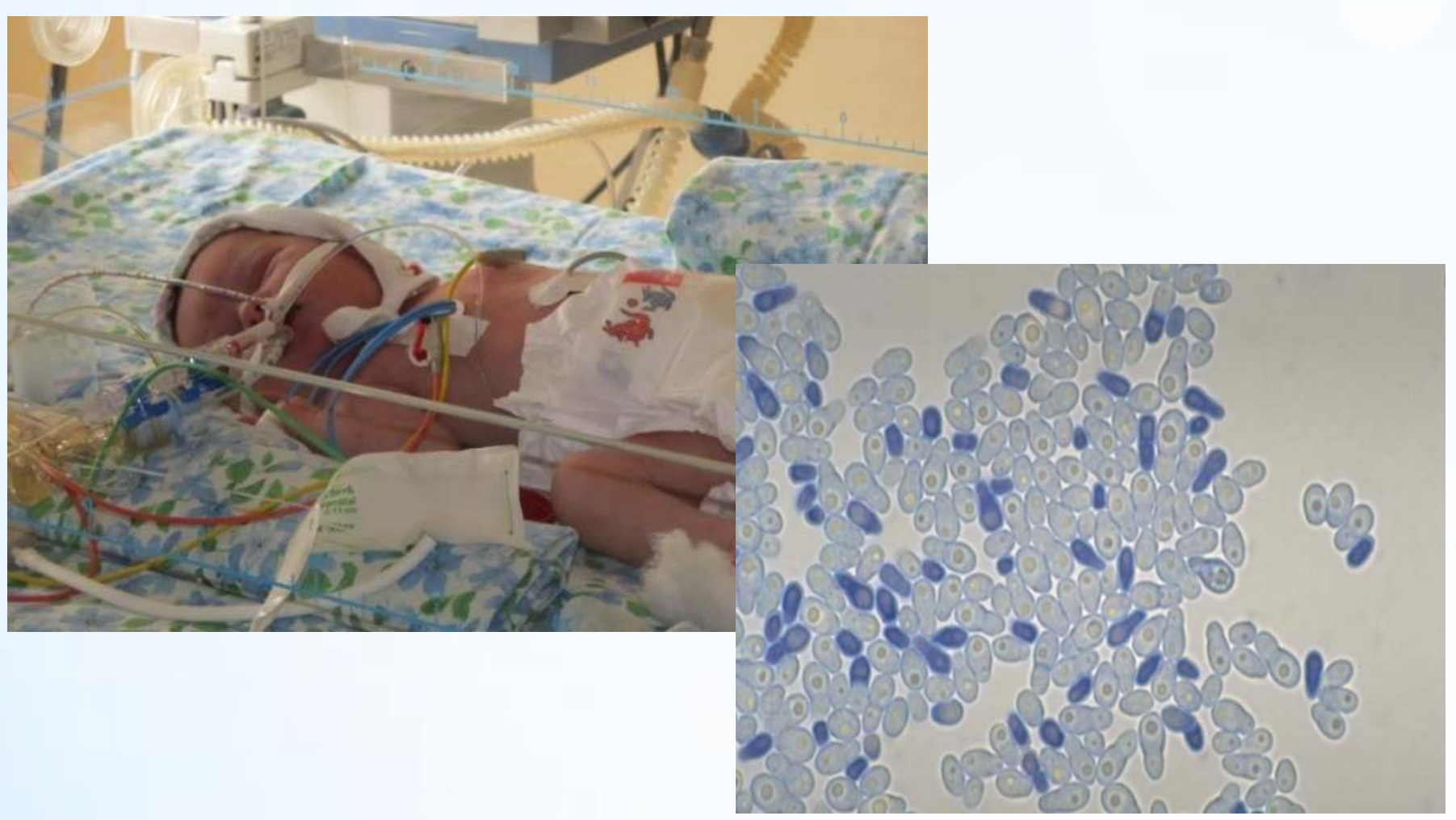

Methods: The study was carried out from 2015 to 2017 and included infants from the neonatal intensive care unit (NICU) and the surgery NICU, all patients were admitted to National Medical Research Center for Obstetrics, Gynecology and Perinatology named after Academician V.I. Kulakov of Ministry of Healthcare of Russian Federation, Moscow. Microbiological monitoring of $M$. furfur included regular pharyngeal, rectal swabs and tracheal tubes once a week from the first day of life. In case of infection signs additional microbiological tests were done (blood, urine and venous catheter). All biological specimens and swabs were cultured on modified Dixon agar for $72 \mathrm{~h}$ at $32{ }^{\circ} \mathrm{C} \pm 2{ }^{\circ} \mathrm{C}$. The yeast identification was based on morphological and biochemical features and using an automatic bacteriological analyzer Vitek 2 Compact (bioMerieux, France). For blood cultivation liquid modified Dixon agar and Bact/Alert (bioMerieux) were used.
Results: From 2015 to 20172793 infants were observed: $70 \%$ (1942 infants) were infants from the NICU and 30\% (851 infants) - from the surgery NICU. M. furfur was found in $14 \%$ of them (293 infants): $41 \%$ of cases were infants from the NICU and 30\% (174 infants) - from the surgery NICU. All observed infants had some risk factors for $M$. furfur colonization: low or very low birth weight, parenteral nutrition by lipid solutions, abdominal or chest operations. Average weight among all infants with $M$. furfur was 2124 grams Average weight among infants from the NICU was 1038 grams. Average weight among infants from the surgery NICU was 2899 grams. Totally 106 newborns (3.8\%) had systemic mycoses (isolation from blood, venous catheters, urine, and autopsy material). The morbidity of fungal infections caused by Malassezia furfur in the NICU was $2.4 \%$ whereas in the surgery NICU the morbidity was $6.9 \%$. During monitoring from feces and pharynx the morbidity was $6.7 \%$ (187infants).
Conclusion: The morbidity among the surgery NICU was $6.9 \%$ and was higher than in the NICU. The risk factors for $M$. furfur colonization in the surgery NICU were parenteral nutrition by lipid solutions and surgical operations. The morbidity among the NICU was $2.4 \%$. The main risk factors for $M$. furfur colonization in the NICU were low or very low birth weight and parenteral nutrition by lipid solutions. 